\title{
Scanned images resolution improvement using neural networks
}

\author{
Electronics Laboratory, Physics Department, University of Patras, Rio 26500, \\ Greece. \\ Communicating author: \\ Vassilis Anastassopoulos \\ Professor \\ Electronics Laboratory \\ Physics Department \\ University of Patras \\ Rio 26500, Greece \\ Tel: +302610 996147 \\ Fax: +302610 997456 \\ Email: vassilis@physics.upatras.gr
}

\begin{abstract}
A novel method of improving the spatial resolution of scanned images, by means of neural networks, is presented in this paper. Images of different resolution, originating from scanner, successively train a neural network, which learns to improve resolution from 25 to 50 pixels-per-inch (ppi), then from 100 to 200 ppi and finally, from 50 to 100 ppi. Thus, the network is provided with consistent knowledge regarding the point spread function (PSF) of the scanner, whilst it gains the generalization ability to reconstruct finer resolution images unfamiliar to it. The novelty of the proposed image-resolution-enhancement technique lies in the successive training of the neural structure with images of increasing resolution. Comparisons with the image scanned at 400 ppi demonstrate the superiority of our method to conventional interpolation techniques.
\end{abstract}

Keywords Resolution improvement·Neural network·Scanner 


\section{Introduction}

Low-resolution (LR) images are commonly found in many imaging applications, such as remote sensing, surveillance and astronomy. Over the last two decades, research has been devoted to the problem of reconstructing a high-resolution (HR) image from a LR frame. Basically, interpolation techniques have been used to improve resolution in medical images [1], digital photos [2], as well as in image sequences (video) [3-4]. In general, interpolation techniques produce a fine resolution image from a given LR image. A great volume of reports on image interpolation using neural networks (NN) can be found in the literature [2-3, 5-17]. The present work, which attempts to obtain a HR image by successively training the same NN, belongs to this category. Neural networks describe systems without an explicit physical and mathematical modeling. Actually, the NN is trained to successfully carry out the solution of inverse problems, which in our case corresponds to restoring effects caused by scanner non-idealities.

Super-resolution techniques constitute a quite different image-processing tool. It is actually the estimation of a single HR image from multiple LR ones with subpixel shifts. The resulting image is much closer to the desired one, compared to that obtained using interpolation techniques [4]. Actually, frequency unfolding is achieved, which resolves details in the image finer than the sensor resolution limit. Neural networks have been used for solving the super-resolution image reconstruction problem [5-6]. Nevertheless, it should be mentioned that from time to time interpolation techniques are referred to as super-resolution, resulting in confusing naming.

Interpolation methods by means of neural networks have been employed for resolution enhancement in the area of remotely sensed images. An approach of spatial resolution improvement of a remotely sensed, LR, thermal infrared image is described in [7]. The resolution improvement is carried out by means of two three-layered, backpropagated NN architectures. Another approach of resolution improvement in remotely sensed images is that of a fully-interconnected NN model [8]. The network used during testing is composed of three layers. Image interpolation has also been carried out by means of radial basis function neural networks in [9]. The hidden layer neurons of the network which is employed to perform interpolation compute Gaussian basis functions. The receptive field widths (standard deviations) of these functions are determined by an adaptive scheme. Furthermore, HR image reconstruction has been successfully carried out using multilayer perceptron networks in [10]. It should be noted that two different backpropagation algorithms are used, whereas a multilayer perceptron network is also employed in [11]. The specific single-hidden-layer neural network, being trained by the backpropagation algorithm, is required to enhance the resolution of diffraction-limited, binary images. Moreover, a high-resolution, multi-neural network, based on the local 
variance is proposed in [12]. This specific network is composed of two neural networks, namely the NN for low local variance and the NN for high local variance. The weighted sum of the two NN outputs represents the enlarged image. A novel image interpolation scheme, using an artificial neural network, is described in [13]. A single frame interpolation algorithm is joined together with an adaptive, linear, single-layer neural network that models the residual errors between the interpolated image and the respective original one. A novel image interpolation algorithm by means of a feedforward neural network, based upon classification, is thoroughly considered in [14]. A HVS-oriented, adaptive interpolation scheme for natural images by means of neural networks is proposed in [15]. Furthermore, a color interpolation technique for a single-chip CCD camera, employing neural networks, is presented in [2]. A Hopfield-network-based algorithm, serving for the resolution enhancement of discrete targets taking up more space than the sample spacing of an image, is dealt with in [16]. Multilayer neural networks have also been used to perform document resizing in [17].

In this paper a technique that performs image interpolation using neural networks is presented. A multilayer feedforward (MLFF) neural network, trained by the backpropagation algorithm, is employed. The scanner provides all the input and output data that the network training requires. Pairs of varying resolution images, obtained through scanner, are used to successively train the network, so that it becomes familiar with the scanner point spread function (PSF). More specifically, the neural network is trained with the purpose of becoming able to reconstruct a finer resolution image, even when its input is presented with images of different scenes from those than that it has been trained for. Indeed, the resulting network improves the resolution of scanned images. Scanner, while recording an image, applies a kind of lowpass filtering to this original high quality image and afterwards, by means of a specific sampling, obtains the desired lower resolution image. In this work the NN applies the inverse procedure of the scanner PSF, to a 200 pixels-per-inch (ppi) resolution image in order to construct the 400 ppi HR image. The proposed method outperforms the bicubic and spline algorithms, which belong to classical interpolation techniques. The experimental results as well as the visual comparisons prove the predominance of the proposed method. Indeed, the inversion of the scanner PSF and generalized image interpolation can be considered equivalent. When performing interpolation at 200 ppi to obtain the higher resolution image of $400 \mathrm{ppi}$, an effort towards getting back missing samples of the image is made. Likewise, the NN, having as input the 200 ppi resolution image, results in the $400 \mathrm{ppi}$ image estimating the image samples which were discarded during this image acquisition. Consistent pieces of information regarding these samples can be obtained from the procedure applied to the HR image by the scanner. The novelty of our approach lies in the 
presentation order of image-pairs with different resolution used to train the neural network. To the authors' knowledge there has not been any similar work in literature so far, examining the importance of resolution order when presenting a neural network with training data.

In Section 2 of this paper, the performance of the scanner as image source is presented. Section 3 consists of a detailed description of the training procedure, while Section 4 deals with the results of the conducted experiment. Section 5 presents some decisive aspects concerning our treatment of the neural structure for the prementioned purpose, whereas conclusions are drawn in Section 6.

\section{Using scanner as image source}

Scanning devices are able to produce images of the same scene characterized by different resolution. The whole procedure that the scanner applies to an image is described by

$$
i_{s}(n, m)=\left\{o(x, y) * g(x, y) * \operatorname{rect}\left[\frac{x}{a}, \frac{y}{b}\right]\right\} \times \operatorname{comb}\left(\frac{x}{x_{s}}, \frac{y}{y_{s}}\right)
$$

where $i_{s}(n, m)$ is the ensemble of the produced detector signals, $o(x, y)$ is the object scene (input signal), $g(x, y)$ is the point spread function of the front optics and $\operatorname{rect}\left(\frac{x}{a}, \frac{y}{b}\right)$ is the detector pupil. The 2-D comb-function $\operatorname{comb}\left(\frac{x}{x_{s}}, \frac{y}{y_{s}}\right)$ describes the scanning process. The symbol * denotes 2-D convolution. Scanner PSF is given by relation (1) if $o(x, y)$ denotes a delta function. In accordance with the typical definition of scan directions, spatial scanning in the direction of the detector line is called in-scan direction, while the orthogonal direction, which is along the direction of scan motion for a 1-D array, is given the name cross-scan. Altogether it represents a 2-D scanning process, which can be assumed to be separable and described in both directions as a 1-D process. During scanning with a single 1-D detector array, the spatial scanning frequency $k_{s}$ is in reverse proportion to the middle distance $p$ of neighboring detector pixels. This spatial sampling frequency $\frac{1}{p}$ is equal to the distance of the delta impulses in the comb-function [18]. During image acquisition, different values can be assigned to the characteristic parameter $p$, resulting in images of different resolution. Thus, the comb-function is the element of equation (1), which takes various values during the recording of different resolution images. 
Moving from a high-resolution image to a lower-resolution one, a group of pixels is replaced by one pixel. In theory, this replacement is determined by the scanner characteristics [18-19]. A large pixel of LR image can be obtained from a group of HR image pixels, as shown in Fig. 1, taking into consideration the scanner PSF and the 2-D sampling theorem. In case that the PSF of the scanner is known, the unique evaluation of the LR image pixel from its HR image counterparts can take place. Nevertheless, the reverse procedure is not possible by means of a single LR image, even if the scanner PSF is known. In our case, the scanner PSF is considered unknown, thus the proposed approach is universal, whereas the resulting neural network succeeds in performing the inverse procedure of the scanner PSF. This leads the network to enhancing the resolution of a given image not having previously been trained upon it.

The reason for creating a neural network model with the aforementioned ability lies in the scanner's capability of providing us with varying resolution images. The use of scanned images in this work provides the advantage of having both the input and output vectors required to train the network from the original source. Otherwise, LR images have to be created from the HR data by means of decimation procedures, an approach that demands knowledge of scanner characteristics [18-19]. In the experimental procedure followed, images of six different scenes are obtained from the scanner and subsequently used in the training and simulation procedures. Changing the above mentioned sampling distance $p$, each scene is recorded at 25, 50, 100, 200 and 400 ppi resolutions.

\section{Training procedure}

A MLFF neural network is employed to perform the image resolution enhancement task. The specific neural architecture is shown in Fig. 2. It is a hierarchical network consisting of an input layer, a hidden layer and an output layer. Each neuron of the input layer is connected through synoptic weights to all the hidden layer neurons. The neurons of the hidden layer are also connected to the four neurons of the output layer in a similar way. Three layers are theoretically sufficient to cope with any application. Usually, the hidden layer possesses the greater number of neurons, as the contribution of these neurons to the successful execution of the desired mapping is considered crucial. Nevertheless, in the present case, experimentation proved that the first two layers should have equal number of neurons. These two layers consist of ten neurons, while the third one of four neurons, as required by the dimensions of the output vector. The neural network performance has been also tested using, at the first and second layers, the following numbers of neurons: five and ten, seven and ten, ten and fifteen, ten and twenty respectively, but the results were poorer compared with those obtained using ten neurons. The backpropagation 
learning method is applied to the network. Like the delta rule, it is an optimization procedure based on gradient descent that adjusts weights to reduce the system error or cost function. The proposed training procedure of the neural network is shown in Fig. 3. The training data consist of four different resolution images, which illustrate the scene "poros" shown in Fig. 4. Specifically, the images "poros" of 25, 50, 100 and 200 ppi resolutions are employed to train the neural structure. These data are successively fed to the network input and output. There is a serial implementation of three training stages. It should be stressed that each training stage deals with increasing resolution images of the scene "poros".

At the first training step, the network is fed with the 25 ppi resolution image and the output consists of the 50 ppi resolution image. Afterwards, the images with 100 and 200 ppi resolution are used to form the network input and output respectively. At the final, third step, the neural network goes through training with the 50 ppi resolution image as input and the 100 ppi resolution image as output. At every one training step, the input image is given per 25-component vectors, while the output image is presented per 4-component vectors. These 25-element vectors are progressively selected from the LR image in such a way that the whole image is covered. As shown in Fig. 1, at the HR image reconstruction, the central pixel of each of these sets is replaced by 4 new pixels coming from the one-step-higher resolution image. Each of the 4-element vectors that corresponds to the neural network output, is used to form the high-resolution image. Thus progressively, all the pixels of the low-resolution image are substituted and this image is transformed to the one-step-higher resolution one. Due to the fact that every pair of images employed during each training stage is made up of increasing resolution images, the neural network is supplied with consistent pieces of knowledge as far as the scanner PSF is concerned. In this way, it is successfully taught how to construct an image characterized by resolution not previously presented to it. The obtained resolution is higher than that of each of the images used as training data. Indeed, the resulting neural network, when presented with a 200 ppi resolution image illustrating whatsoever scene, produces a satisfactory image of 400 ppi resolution.

The neural network is not reset from one training stage to another. Thanks to it, knowledge transfer regarding resolution improvement takes place from stage to stage. The resolution order of the training data presentation to the network is another decisive point. The suggested presentation of the images results in an efficient learning procedure. Furthermore, the MSE obtained during training is of order $10^{-2}$. Nevertheless, it is of crucial importance that the network acquires satisfactory generalization ability. The generalization ability is related to the successful reconstruction of a $400 \mathrm{ppi}$ resolution image, when the specific network is simulated with a 200 ppi resolution image. The 
reconstruction should be successful not only in terms of the image "poros", as being the network input, but of various scenes images as well. As it is shown below, the neural network obtains the desired generalization ability.

\section{Experimental results}

After the training procedure has been completed, the obtained neural network goes through the simulation procedure. The image "poros" is used for training the neural network. During simulation, five unfamiliar to the network images, illustrating various scenes of resolution $200 \mathrm{ppi}$, are successively presented to its input along with the image "poros" of the same resolution. One of these new images, the image "pylos", is shown in Fig. 5. Images of 400 ppi resolution, which prove satisfactory when compared with the corresponding scanner-originated ones, are created at the neural network output. Additionally, the trained neural structure is tested with noisy data and the results are in favor of the proposed method.

\subsection{Numerical and visual comparisons}

Besides visual comparison, our method is also assessed by means of mean square error (MSE), mean and standard deviation values in comparison with the bicubic and spline interpolation algorithms. Tables I and III report on these estimated values as far as the image "poros" is concerned. The proposed serial training procedure leads the neural network to a 400 ppi resolution image that demonstrates MSE $=0.0405$. This value is lower than the MSE $=0.0433$ evaluated for the images resulting from the bicubic and spline algorithms. Moreover, the mean value of the network-produced image is equal to 0.5263 and approximates satisfactorily the original image one, which is equal to 0.5340 . Images coming from the bicubic and spline methods display mean values 0.5200 and 0.5034 respectively, which are quite distant from the mean value of the scanneroriginated image. Furthermore, the standard deviation value, equal to 0.2329 , of the network-produced image is quite close to the desired value of 0.2615 in contradiction with the values 0.2103 and 0.1921 of the bicubic and spline algorithms images.

Additionally, the obtained neural network demonstrates satisfactory results when it is simulated with images that have not been used during its training procedure. Tables II and III contain numerical results for the image "pylos". The 0.0409 MSE of our method is quite lower than the 0.0428 and 0.0424 errors of the bicubic and spline interpolations. Furthermore, the mean and standard deviation values of the image obtained by the proposed method, approach the original values more efficiently compared with those of 
the images obtained by means of the two classical interpolation methods. Visual comparison, Fig. 4 and 5, also proves the predominance of the proposed method. Comparison results regarding the rest four images used during simulation have shown to conclude in favor of the proposed method, too.

From the above analysis it is obvious that the image resolution improvement carried out by means of our neural network outperforms the corresponding results of the spline and bicubic interpolation algorithms. It should be stressed that the proposed method leads to a neural network, which is not specialized in improving the resolution of a specific scene image. The employed neural structure, through the presented training procedure, learns how to improve image resolution and thus successfully constructs HR images not having previously been trained upon. All the MSEs are evaluated using the 400 ppi resolution image coming from the scanner. It is noted that the range of the pixel values is $0-1$, whereas the programming tool used is MATLAB.

\subsection{Edge details assessment}

In order to provide a more detailed assessment of the proposed method a small part of the image "poros", shown in Fig. 6, is acquired by means of the scanner at 25, 50, 100, 200 and 400 ppi resolutions. This specific scene image is then used to train the neural network of Fig. 2. Its simulation with the 200 ppi resolution image leads to the image of $400 \mathrm{ppi}$ resolution illustrated in Fig. 6a. The corresponding result obtained by means of the bicubic method is shown in Fig. 6b. Visual comparison proves the predominance of the proposed method as far as edge preserving is concerned. Our neural network achieves detailed and exact reconstruction, while the conventional interpolation technique is not satisfactorily edge preserving. The outline of the small window cited just above the door next to the white car and the right side of the black door coming immediately after the dark coloured car indicate that the proposed method is more capable of retaining edges and avoiding the introduction of even small artifacts in comparison with the bicubic algorithm. The above result is quantified via the edge correspondence measure. Prewitt edge detector is used to find edges in the 400 ppi image of Fig. 6a, in the corresponding image coming from the bicubic method and in the original small part of the image poros originating from the scanner. Then, the number of correct edges that the images of Fig. 6a and $6 \mathrm{~b}$ present is calculated. The image of the proposed method has 68 correct edges, while the image obtained by means of the bicubic algorithm presents 44 correct edges. The spline algorithm outcome is not presented here but is the same as that of the bicubic algorithm. 


\subsection{Performance under noisy conditions}

Furthermore, the performance of the obtained neural network under noisy conditions is examined. Gaussian noise with variance 25 is added to the image "poros" of 200 ppi resolution, which is then fed to the network input. This degraded image is shown in Fig. 7a. The 400 ppi resolution image produced at the network output is depicted in Fig. 7b. Figures $7 \mathrm{c}$ and $7 \mathrm{~d}$ illustrate the result of creating a 400 ppi resolution image from the degraded 200 ppi resolution image, by means of the bicubic and spline interpolation algorithms. It is visually obvious that the proposed method outperforms the aforementioned two conventional interpolation techniques. The neural network results in an image with noise almost eliminated, in contradiction with the images coming from the bicubic and spline interpolation algorithms.

\subsection{Computational details}

The neurons of the first two layers use the tan-sigmoid transfer function to generate their outputs, while the activation function of the last layer neurons is the log-sigmoid transfer function. The Powell-Beale version of the conjugate gradient algorithm is the selected training function. Furthermore, the training parameter concerning the magnitude of the gradient is set to the value $10^{-5}$, so that it can be never reached. As far as the learning parameter is concerned, it is set equal to $10^{-2}$. The number of training epochs for each stage is 250. Each output image reconstruction requires the estimation of $\left(n_{1}-4\right) \times\left(n_{2}-4\right)$ 4-component vectors, where $n_{1}, n_{2}$ denote the number of the input image pixels in the vertical and horizontal directions, respectively. The calculation of each of the above 4-component vectors is performed by a neural network that functions having $25 \times 10+10 \times 10+10 \times 4=390$ weights. The number of coefficients used by the bicubic interpolation algorithm is 13 , while the spline interpolation uses 361 coefficients. Thus, the computational complexity of our neural-network-based method is of the same order as that of the spline interpolation, but of higher order than that of the bicubic interpolation.

\subsection{Remarks}

It should be mentioned that during the training procedure the neural network may reach a specific value of training MSE but cannot proceed to a lower level. If it is forced by the number of training epochs to repeatedly reach this MSE value, due to continuous training, it becomes unable to fulfill our expectations concerning generalization ability. More 
clearly, overtraining occurs because the neurons memorize the training set patterns and become unable to recognize target-class patterns that were not included in the training set. Thus, using a number of epochs larger than the 250 selected is not recommended. In contrast, fewer than 250 training epochs are inadequate for efficient learning of the neural network. Overtraining also takes place in case of using more than ten neurons in the first two layers, whereas fewer than ten neurons are insufficient in view of our purpose. Additionally, when choosing a value of the learning rate parameter higher than $10^{-2}$, the whole procedure may not converge to the appropriate network weight values [20]. Numerous combinations of activation functions used by the neural network neurons have also been tested. This aforementioned combination tansig-tansig-logsig was the most efficient. Moreover, the presentation order of training data to the neural network has proved to be of great significance, as well. The serial training procedure has also been tested, with input-output image pairs 25-50, 50-100 and 100-200 ppi. However, the obtained network appears to possess limited abilities. Furthermore, various combinations of the prementioned three training stages for the implementation of a nine-stagesrepetitive training procedure have been examined. Nevertheless, this repetitive implementation did not prove efficient as the resulting image was over-smoothed.

\section{Discussion}

The fact that the neural network weights are not reinitialized from training step to training step on the one hand, and the order the training images are fed to the network on the other, are two decisive points of the presented strategy. Both result in retaining the neural network knowledge from one training stage to another. An additional significant point is the neural structure training with images coming directly from the scanner. Due to the originality of this training procedure, the network is provided with the appropriate pieces of knowledge, so that it can learn to improve image resolution. Moreover, when treating a neural network special attention should be paid to avoid overtraining. The number of the training epochs as well as the number of the neurons in each layer must be carefully selected. It should be stressed that the low value of the MSE obtained during training does not keep pace with a visually satisfactory image of 400 ppi derived from the network, as it differs from the adequate generalization ability. This ability is indeed connected to the desired, successful reconstruction of the HR image. Nonetheless, it should be stated that the visual inspection remains the most reliable measure of comparison. Moreover, it is evident that neural structures cannot easily retain the knowledge gained by means of a specific set of training data when a new training data set is presented to them. Furthermore, during the training procedure the network employed 
for interpolation is provided with consistent knowledge of the standard deviation and mean values of the image of finer resolution. In reverse, when creating an interpolated image through the bicubic and spline methods, there are no a priori pieces of knowledge of these characteristic values of the constructed image.

\section{Conclusions}

In this work, a multilayer feedforward neural network is employed to improve the resolution of scanned images. Pairs of different resolution images, illustrating the scene "poros" and coming from the scanner, are used in the training procedure. There are three training stages each of which deals with a pair of increasing resolution images. The neural network gains knowledge concerning resolution enhancement from 25 to 50 ppi, from 100 to $200 \mathrm{ppi}$ and from 50 to $100 \mathrm{ppi}$. In this way it achieves to efficiently learn the PSF of the scanner. The rationale behind the proposed approach is the training of the network in order not only to learn how to improve a specific image resolution to a finer one, but to generalize this knowledge to even finer resolution and different scene image, as well. Actually, the suggested successive presentation of the different resolution training data to the network has led to the successful application of the inverse procedure of the scanner PSF to 200 ppi resolution images that are unfamiliar to it. Satisfactory images of $400 \mathrm{ppi}$ resolution illustrating even different scenes from the scene "poros" are the outcome of this attempt. Visual comparisons, MSE, mean and standard deviation values have proved that the proposed method predominates over the classical bicubic and spline interpolation algorithms. This method reconstructs images of 400 ppi resolution that successfully approach the corresponding original images obtained by means of the scanner. The proposed method also performs superiorly even under noisy conditions. The present method is novel as it treats, for the first time, the issue of the training data presentation order to the neural network input.

\section{References}

1. Lehmann T M, Gonner C, Spitzer K (1999) Survey: Interpolation Methods in Medical Image Processing. IEEE Trans on Med Imag 18:1049-1075.

2. Go J, Sohn K, Lee Ch (2000) Interpolation using neural networks for digital still cameras. IEEE Trans on Consum Electr 46:610-616.

3. Skoneczny S, Szostakowski J (2001) Classical and neural methods of image sequence interpolation. Proc of SPIE 4535:191-204. 
4. Tekalp A M (1995) Digital video processing. Prentice-Hall, United States of America

5. Salari E, Zhang S (2003) Integrated recurrent neural network for image resolution enhancement from multiple image frames. IEE Proc 150:299-305.

6. Tsagaris V, Panagiotopoulou A, Anastassopoulos V (2004) Interpolation in multispectral data using neural networks. Proc of SPIE 5573:460-470.

7. Valdes M del C, Inamura M (1998) Spatial Resolution Improvement of a Low Spatial Resolution Thermal Infrared Image by Backpropagated Neural Networks. IEICE Trans Inf \& Syst E81-D:872-880.

8. Valdes M del C, Inamura M (2000) Spatial Resolution Improvement of Remotely Sensed Images by a Fully Interconnected Neural Network Approach. IEEE Trans on Geosc and Rem Sens $38: 2426-2430$.

9. Ahmed F, Gustafson S C, Karim M A (1996) Image interpolation with adaptive receptive fieldbased Gaussian radial basis functions. Microw and Opt Techn Lett 13:197-202.

10. Plaziac N (1999) Image Interpolation Using Neural Networks. IEEE Trans on Imag Process 8: 1647-1651.

11. Davila C A, Hunt B R (2000) Superresolution of binary images with a nonlinear interpolative neural network. Appl Opt 39:2291-2299.

12. Sekiwa D, Taguchi A (2001) Enlargement of Digital Images by Using Multi-Neural Networks. Electr and Commun in Jap 84:61-69.

13. Pan F, Zhang L (2003) New image super-resolution scheme based on residual error restoration by neural networks. Opt Eng 42:3038-3046.

14. Hu H, Hofman P M, Haan G (2004) Image Interpolation Using Classification-based Neural Networks. IEEE Intern Symp on Consum Electr 1:133-137.

15. Pu H-C, Lin C-T, Liang S-F, Kumar N (2003) A Novel Neural-Network-Based Image Resolution Enhancement. IEEE Intern Conf on Fuzz Syst 2:1428-1433.

16. Collins M, Jong M (2004) Neuralizing Target Superresolution Algorithms. IEEE Geosc and Rem Sens Lett 1:318-321.

17. Ahmed M N, Cooper B E, Love S T (2001) Document Resizing Using a Multi-Layer Neural Network. NIP17: Intern Conf on Dig Print Techn:792-796.

18. Craubner S (2002) Optoelectronic image scanning with high spatial resolution and reconstruction fidelity. Opt Eng 41:381-394.

19. Shen H-L, Xin J H (2004) Spectral characterization of a color scanner by adaptive estimation. J Opt Soc Am 21:1125-1130.

20. Patterson D W (1996) Artificial Neural Networks: Theory and Applications. Prentice-Hall, Singapore. 


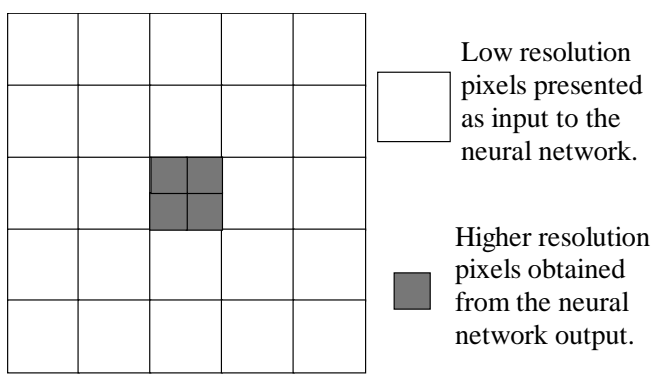

Fig. 1. The arrangement of pixels used to train the neural network. The white pixels come from the low-resolution image whereas the black-shaded ones originate from the one-step-higher resolution image. At the simulation procedure the aforementioned four pixels are created at the network output.

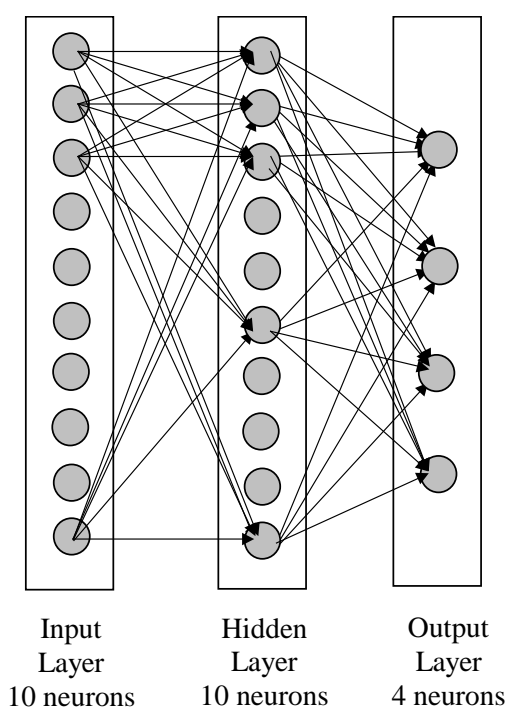

Fig. 2. The neural network architecture used at the proposed interpolation approach. It is a fully interconnected structure, with all outputs from the first layer (left) going to all neurons of the second (hidden) layer. In the same way, all outputs from the second (hidden) layer are fed to all four neurons of the output (third) layer. Each neuron of the input layer is fed with the values of the 25 white pixels shown in Figure 1. 


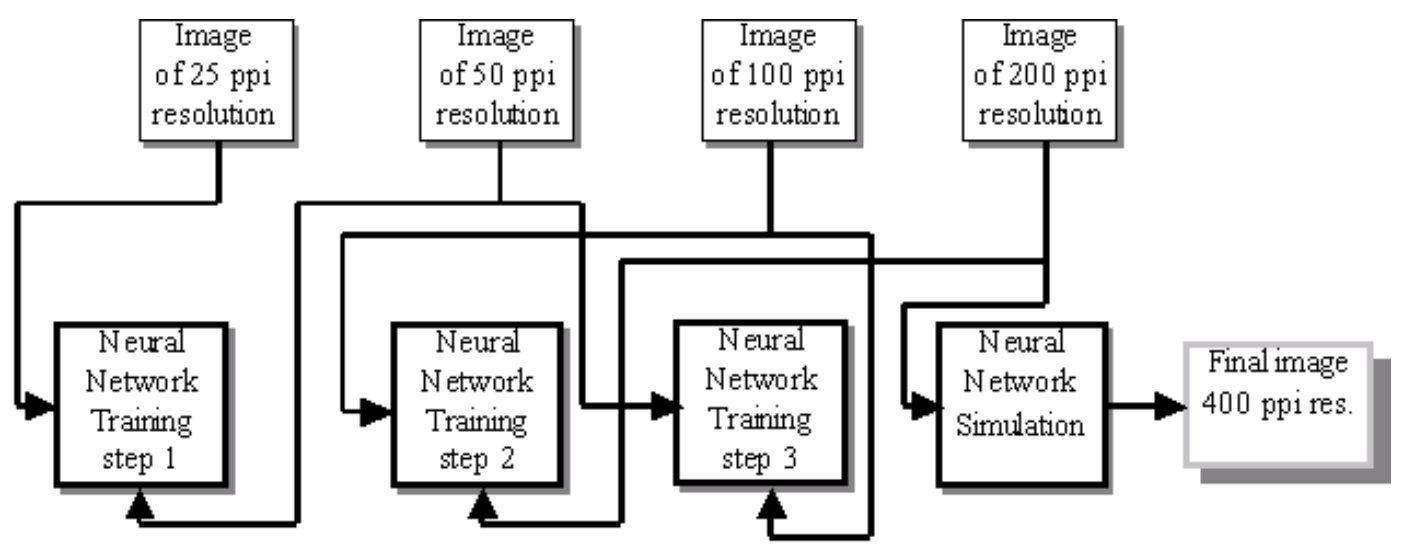

Fig. 3. The proposed method of creating a HR image is based on the successive training of the same neural network with different resolution images.

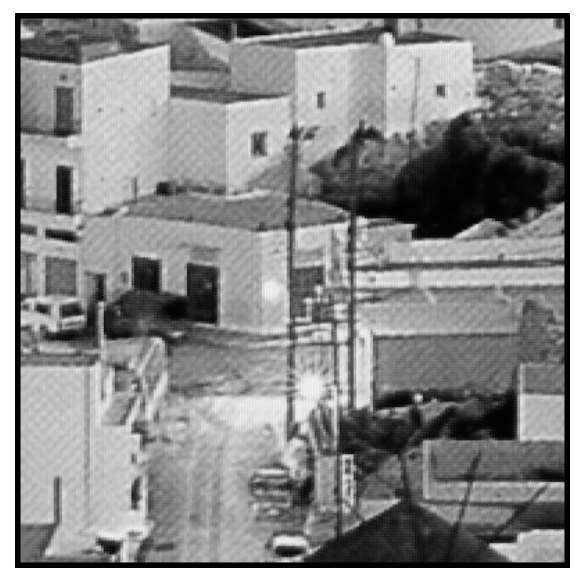

$\mathrm{a}$

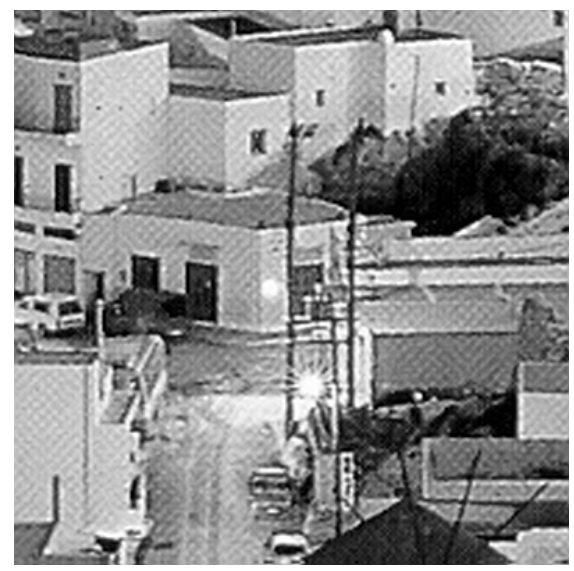

C

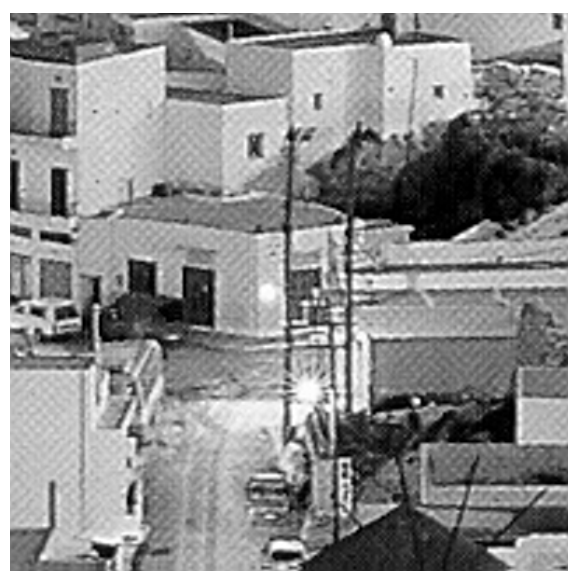

$\mathrm{b}$

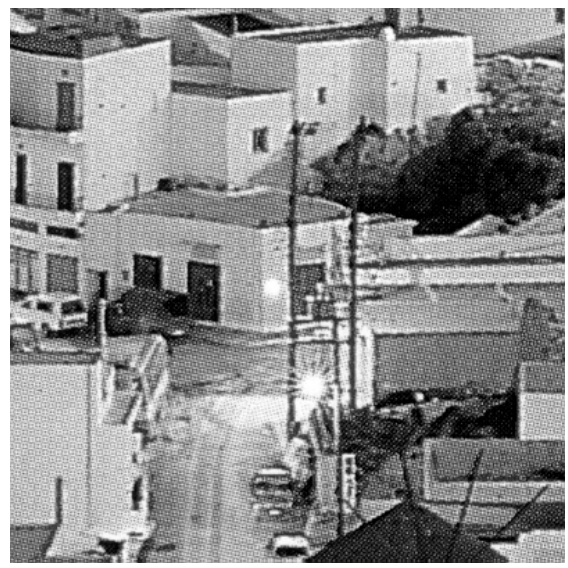

d

Fig. 4. (a) The image "poros" of 400 ppi resolution coming from the neural network. (b) The image "poros" of 400 ppi resolution obtained using the bicubic algorithm. (c) The image "poros" of 400 ppi resolution obtained using the spline algorithm. (d) The image "poros" of 400 ppi resolution coming from the scanner. 


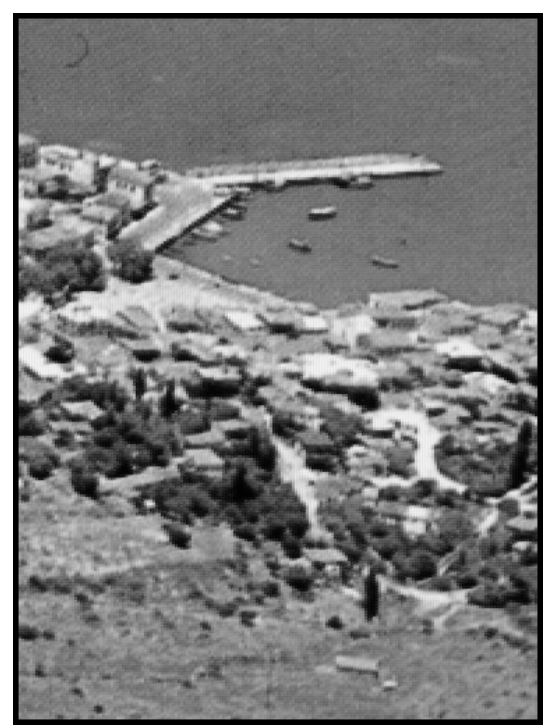

a

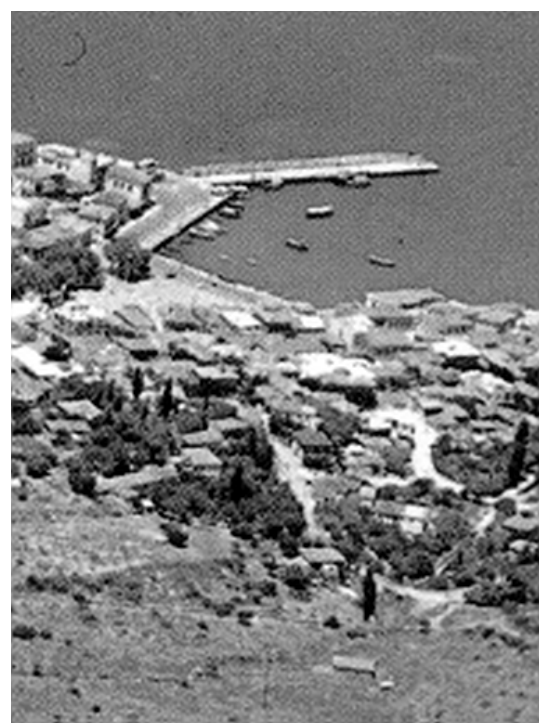

C

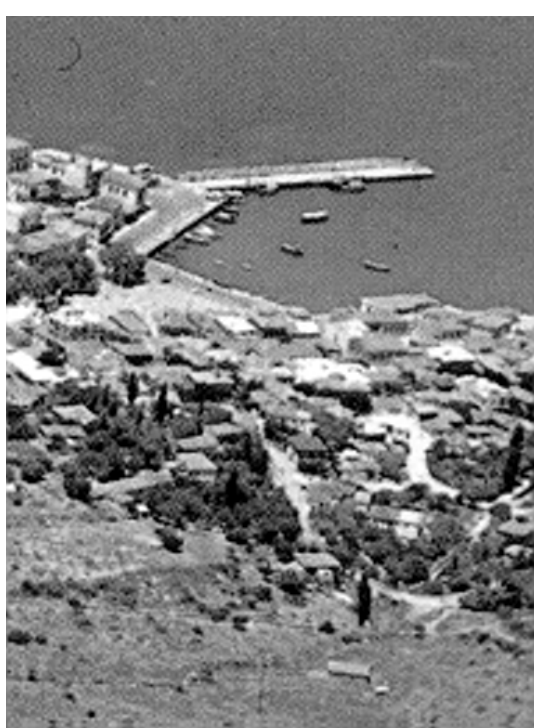

$\mathrm{b}$

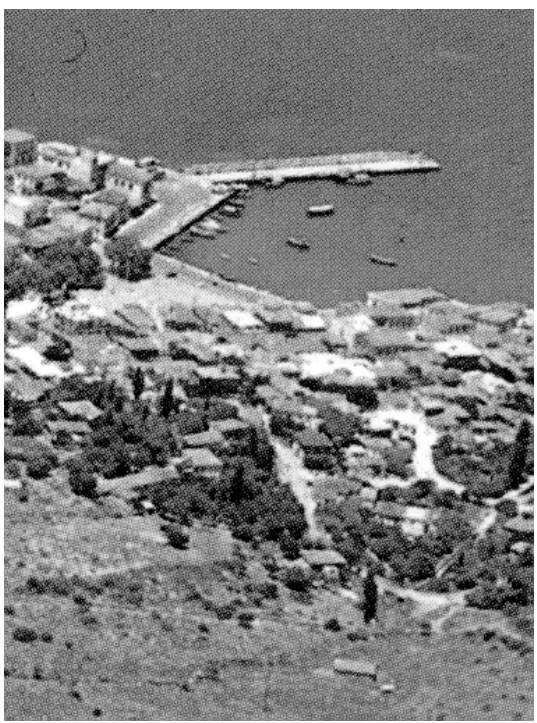

d

Fig. 5. (a) The image "pylos" of 400 ppi resolution coming from the neural network. (b) The image "pylos" of 400 ppi resolution obtained using the bicubic algorithm. (c) The image "pylos" of 400 ppi resolution obtained using the spline algorithm. (d) The image "pylos" of 400 ppi resolution coming from the scanner. 


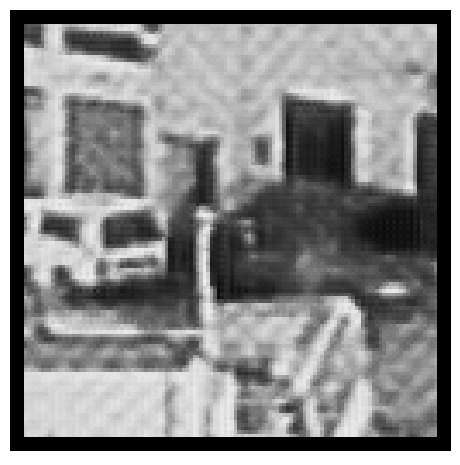

a

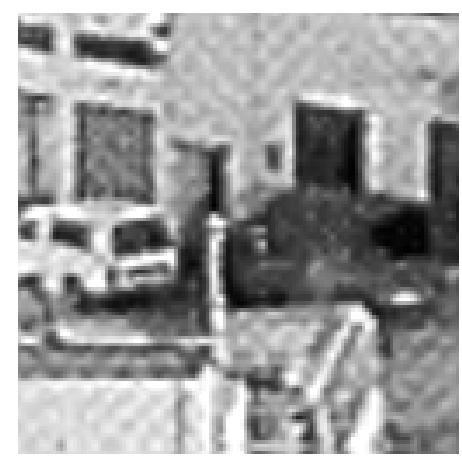

b

Fig. 6. (a) A small part of the image "poros" of 400 ppi resolution coming from the neural network. (b) A small part of the image "poros” of 400 ppi resolution obtained using the bicubic algorithm. It is obvious that the proposed method outperforms the bicubic interpolation as far as edge preserving is concerned. 


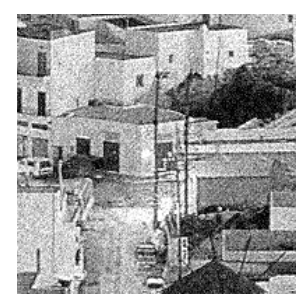

a

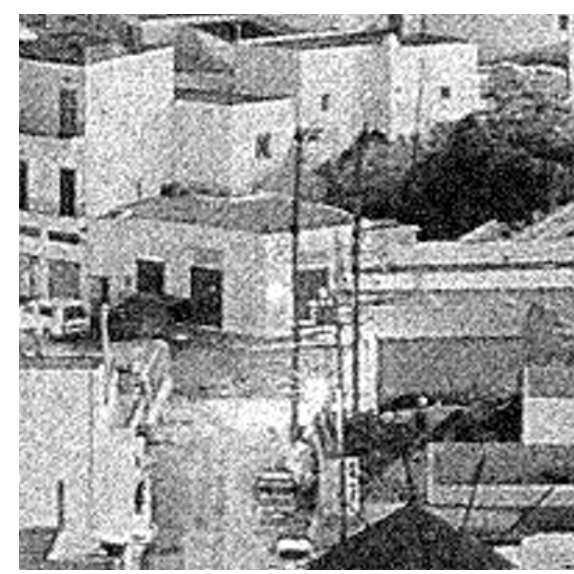

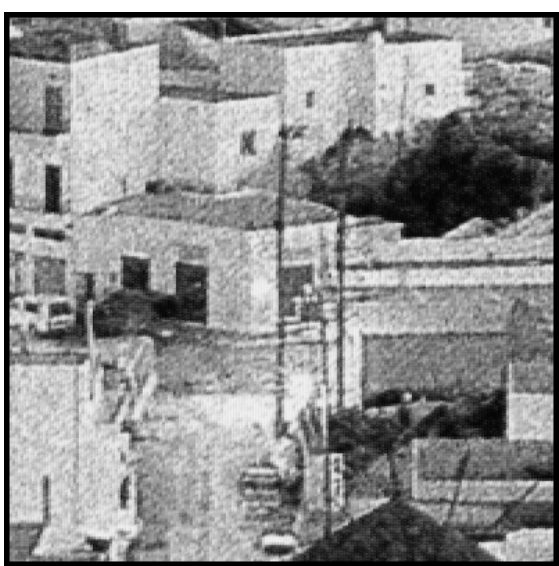

b

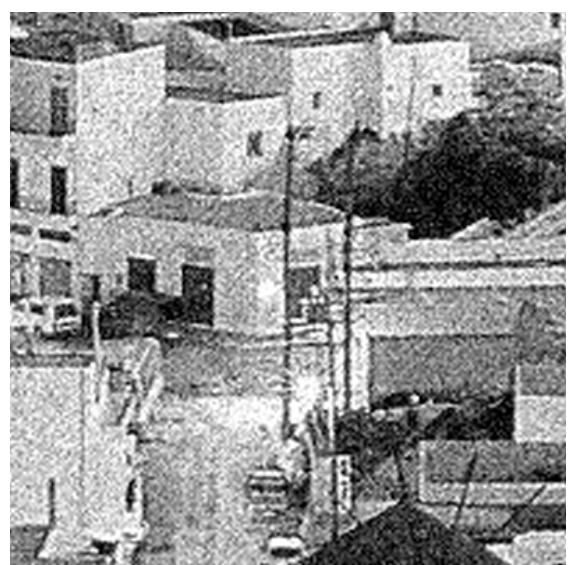

d

Fig. 7. (a) The image "poros" of 200 ppi resolution degraded by Gaussian noise. (b) The image "poros" of 400 ppi resolution coming from the neural network when it is presented with the degraded 200 ppi resolution image. (c) The image “poros” of 400 ppi resolution obtained when performing bicubic interpolation at the degraded 200 ppi resolution image. (d) The image "poros" of 400 ppi resolution obtained when performing spline interpolation at the degraded $200 \mathrm{ppi}$ resolution image. 
Table I: Results in terms of mean and standard deviation values for the image "poros" of $400 \mathrm{ppi}$ resolution

\begin{tabular}{|c|c|c|}
\hline Image Origin & Mean value & Standard deviation value \\
\hline Network output & 0.5263 & 0.2329 \\
\hline Bicubic & 0.5200 & 0.2103 \\
\hline Spline & 0.5034 & 0.1921 \\
\hline Scanner & 0.5340 & 0.2615 \\
\hline
\end{tabular}

Table II: Results in terms of mean and standard deviation values for the image "pylos" of 400 ppi resolution

\begin{tabular}{|c|c|c|}
\hline Image Origin & Mean value & Standard deviation value \\
\hline Network output & 0.4773 & 0.1743 \\
\hline Bicubic & 0.4976 & 0.1684 \\
\hline Spline & 0.5066 & 0.1594 \\
\hline Scanner & 0.4844 & 0.2207 \\
\hline
\end{tabular}

Table III: Results in terms of MSE for the images "poros" and "pylos" of 400 ppi resolution

\begin{tabular}{|c|c|c|}
\hline \multirow{2}{*}{ Real output origin } & \multicolumn{2}{|c|}{ MSE between real output and target output } \\
\cline { 2 - 3 } & “poros” & "pylos” \\
\hline Network output & 0.0405 & 0.0409 \\
\hline Bicubic & 0.0433 & 0.0428 \\
\hline Spline & 0.0433 & 0.0424 \\
\hline
\end{tabular}

